\title{
Why sharks have no bones
}

Elephant shark's genome - the first of a cartilaginous fish - exposes early evolution of vertebrates.

\section{Brendan Borrell}

08 January 2014

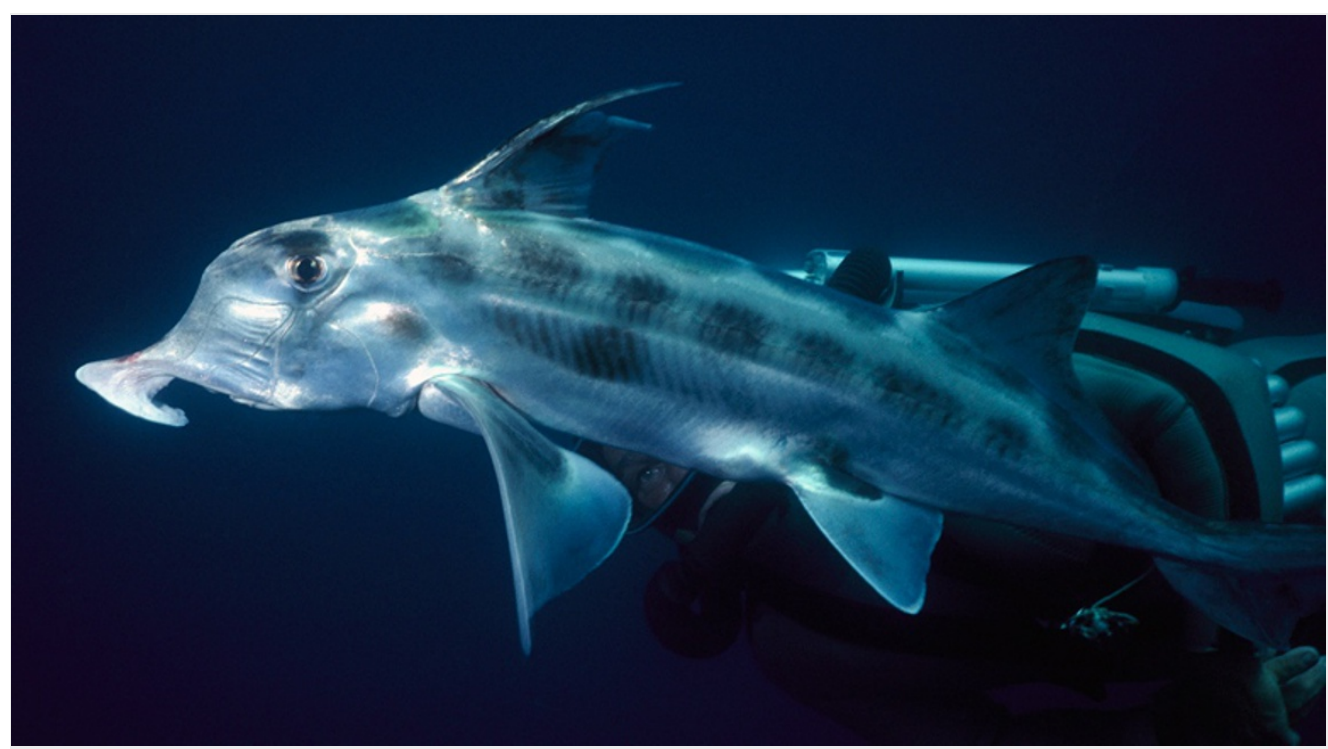

Norbert Wu/Minden Pictures/Getty Images

The elephant shark has changed little in the last 420 million years, making its DNA sequence valuable for comparison with other vertebrate species.

A funky-looking fish with an outsized snout has become the most primitive jawed vertebrate to have its genome sequenced. The DNA sequence of the elephant shark helps to explain why sharks have a cartilaginous skeleton and how humans and other vertebrates evolved acquired immunity.

Elephant sharks (Callorhinchus milii) are part of an early evolutionary branch of cartilaginous fishes known as chimaeras, which are related to sharks and rays. They patrol the deep waters off southern Australia and New Zealand, and use their distinctive snouts to hunt for shellfish buried in the sand. Although elephant sharks are not known to attack humans, they sport a seven-centimetre-long spike on their dorsal fin, which is used to defend against predators.

Six years ago, scientists singled out $C$. milii as the first cartilaginous fish to be sequenced because of its relatively small genome about one-third the size of the human genome. "We have had many genomes for amphibians, for birds and for mammals, but no sharks," says study author Byrappa Venkatesh, a comparative-genomics expert at the Agency for Science, Technology and Research in Singapore.

\section{Dumbo of the sea}

Because the elephant shark is an early jawed vertebrate and has changed little since bony fishes appeared around 420 million years ago — making it the slowest-evolving of all known vertebrates — it serves as an important baseline for comparative genomics. "We are going to use this as a reference for years to come," Venkatesh says. The genome was published today in Nature ${ }^{1}$.

So far, scientists have sequenced the genomes of eight bony fish and two jawless vertebrates known as lampreys. Sharks, skates, rays and chimaeras stand apart from other jawed vertebrates in having a skeleton that is made primarily of cartilage rather than bone. Although scientists knew what genes were involved in bone formation, it wasn't clear whether sharks had lost their bone-forming ability or just never had it in the first place. After all, sharks do make bone in their teeth and fin spines.

The sequence reveals that members of this group are missing a single gene family that regulates the process of turning cartilage into bone, and that a gene duplication event gave rise to the transformation in bony vertebrates. In fact, when the researchers knocked out one of these same genes in a zebrafish, it significantly reduced its ability to form bone. 
John Postlethwait, a developmental biologist at the University of Oregon in Eugene, calls the findings "illuminating". He studies Antarctic icefish (Notothenioidei), which lost the ability to form bone over the course of evolution, and will look to see whether they lack the same genes that are missing from the elephant shark genome.

\section{Evolving immunity}

The C. milii genome also helps to answer important questions about the evolution of acquired immunity, which is the basis for vaccination and allows humans and other vertebrates to fight off new pathogens. Elephant sharks have killer T cells, which directly destroy body cells that have been infected by viruses, but they lack helper T cells, which help to regulate the overall immune response to an infection. The new sequence data suggest that acquired immunity evolved in a two-step process rather than in one step as previously thought.

Efforts are now under way to sequence more cartilaginous fishes, including the little skate (Leucoraja erinacea) from North America and the small-spotted catshark (Scyliorhinus canicula).

Igor Schneider, a evolutionary biologist at the Federal University of Pará, Brazil, who studies how limbs evolved from fish fins, is excited about using the sequence data in his own work. "The elephant shark genome provides an invaluable tool for comparative studies," he says, and he hopes it will help him to determine "the genetic steps towards life on land".

Nature | doi:10.1038/nature.2014.14487

\section{References}

1. Venkatesh, B. et al. Nature 505, 174-179 (2014). 